\title{
A phase II study of preoperative chemotherapy with docetaxel, cisplatin, and S-1 followed by gastrectomy with D2 plus para- aortic lymph node dissection for gastric cancer with extensive lymph node metastasis: JCOG1002
}

\author{
Seiji Ito ${ }^{1}$ ' Takeshi Sano ${ }^{2} \cdot$ Junki Mizusawa $^{3} \cdot$ Daisuke Takahari $^{4} \cdot$ Hiroshi Katayama $^{5}$ • \\ Hitoshi Katai $^{6}$ - Yoshiyuki Kawashima ${ }^{7} \cdot$ Takahiro Kinoshita $^{8} \cdot$ Masanori Terashima $^{9}$ • \\ Atsushi Nashimoto $^{10} \cdot$ Mikihito Nakamori $^{11} \cdot$ Hiroaki Onaya ${ }^{12} \cdot$ Mitsuru Sasako $^{13}$
}

Received: 30 January 2016/ Accepted: 23 May 2016/Published online: 14 June 2016

(c) The International Gastric Cancer Association and The Japanese Gastric Cancer Association 2016

\begin{abstract}
Background Gastric cancer with extensive lymph node metastasis is commonly considered unresectable, with a poor prognosis. We previously reported the results of the use of cisplatin and S-1 as preoperative chemotherapy for gastric cancer with extensive lymph node metastasis; docetaxel, cisplatin, and S-1 (DCS) have now been investigated for the same purpose.

Methods Patients received two or three 28-day cycles of DCS therapy (docetaxel at $40 \mathrm{mg} / \mathrm{m}^{2}$ and cisplatin at $60 \mathrm{mg} / \mathrm{m}^{2}$ on day $1, \mathrm{~S}-1$ at $40 \mathrm{mg} / \mathrm{m}^{2}$ twice daily for 2 weeks) followed by gastrectomy with D2 plus para-aortic nodal dissection. After R0 resection, S-1 chemotherapy was given for 1 year. The primary end point was the response rate (RR) to preoperative chemotherapy determined by central peer review according to the Response Evaluation Criteria in Solid Tumors version 1.0. The
\end{abstract}

On behalf of the Stomach Cancer Study Group of the Japan Clinical Oncology Group.

Seiji Ito

seito@aichi-cc.jp

1 Department of Gastroenterological Surgery, Aichi Cancer Center Hospital, 1-1 Kanokoden, Chikusa-ku, Nagoya 464-8681, Japan

2 Department of Surgery, Cancer Institute Hospital of Japanese Foundation for Cancer Research, Tokyo, Japan

3 JCOG Data Center, National Cancer Center, Tokyo, Japan

4 Department of Gastroenterology, Cancer Institute Hospital of Japanese Foundation for Cancer Research, Tokyo, Japan

5 JCOG Operations Office, National Cancer Center, Tokyo, Japan

6 Gastric Surgery Division, National Cancer Center Hospital, Tokyo, Japan planned sample size was 50, with one-sided alpha of $10 \%$, power of $80 \%$, expected RR of $80 \%$, and threshold of $65 \%$.

Results Between July 2011 and May 2013, 53 patients were enrolled, of whom 52 were eligible. The clinical RR was $57.7 \%$ [30/52, $80 \%$ confidence interval $47.9-67.1 \%$, $p=0.89]$, and R0 resection was achieved in $84.6 \%$ of patients (44/52). Common grade 3 or grade 4 adverse events during DCS therapy were leukocytopenia (18.9\%), neutropenia $(39.6 \%)$, and hyponatremia $(15.1 \%)$. The common grade 3 or grade 4 surgical morbidity was abdominal infection $(10.2 \%)$. The pathological RR was $50.0 \%(26 / 52)$.

Conclusions Preoperative DCS therapy was feasible but did not show a sufficient RR. Preoperative cisplatin and S-1 therapy is still considered the tentative standard treatment for this population until survival results are known.

7 Division of Gastroenterological Surgery, Saitama Cancer Center, Saitama, Japan

8 Gastric Surgery Division, National Cancer Center Hospital East, Kashiwa, Japan

9 Division of Gastric Surgery, Shizuoka Cancer Center, Nagaizumi, Japan

10 Department of Surgery, Niigata Cancer Center Hospital, Niigata, Japan

11 Second Department of Surgery, School of Medicine, Wakayama Medical University, Wakayama, Japan

12 Department of Diagnostic Radiology, Gunma Prefectural Cancer Center, Ota, Japan

13 Division of Upper Gastrointestinal Surgery, Department of Surgery, Hyogo College of Medicine, Nishinomiya, Japan 
Keywords Adjuvant chemotherapy - Gastrectomy · Lymphatic metastasis - Stomach neoplasms

\section{Introduction}

Gastric cancer is the fifth commonest form of cancer and the third commonest cancer-related cause of death in the world [1]. Surgical resection is a vital part of curative treatment for most patients with localized disease, such as regional lymph node metastasis. Gastric cancer with extensive lymph node metastasis (ELM), which is defined as para-aortic lymph node (PAN) metastasis or bulky lymph nodes along the celiac, splenic, common, or proper hepatic arteries, or both of them, is commonly regarded as unresectable. Park et al. [2] reported favorable outcomes of gastric cancer patients with isolated PAN involvement with palliative chemotherapies, but the 3-year overall survival for patients with de novo PAN metastasis was only $13.1 \%$. Even when the tumor can be resected, the prognosis of patients with ELM is poor. According to data collected by the Stomach Cancer Study Group (SCSG) of the Japan Clinical Oncology Group (JCOG), the 3-year survival of 86 patients with both clinical and pathological PAN metastasis who underwent surgery was only $5 \%$. The SCSG/JCOG has developed a preoperative chemotherapy approach as a multidisciplinary treatment strategy for gastric cancer patients with ELM.

Since 2000 we have conducted two phase II trials (JCOG0001 and JCOG0405) to evaluate the safety and efficacy of preoperative chemotherapy followed by gastrectomy with D2 plus PAN dissection for gastric cancer with ELM. In JCOG0001, patients received two or three cycles of irinotecan $\left(70 \mathrm{mg} / \mathrm{m}^{2}\right.$ on days 1 and 15) and cisplatin $\left(80 \mathrm{mg} / \mathrm{m}^{2}\right.$ on day 1$)$ therapy, followed by surgery. This study showed a good 3-year survival of $27.0 \%$, but it was terminated because of three treatment-related deaths among the 55 enrolled patients [3]. Thereafter, in JCOG0405, patients satisfying the same eligibility criteria as in JCOG0001 received two or three cycles of cisplatin $\left(60 \mathrm{mg} / \mathrm{m}^{2}\right.$ on day 8$)$ and S- $1\left(40 \mathrm{mg} / \mathrm{m}^{2}\right.$ twice daily from day 1 to day 21 followed by a 1 -week rest period) (CS) chemotherapy and then underwent surgery. This study showed an excellent response rate (RR) of $64.7 \%$ and a 3 -year survival of $58.8 \%$, with no treatment-related deaths and good feasibility [4]. Since then, preoperative CS chemotherapy has been regarded as the current tentative standard treatment for gastric cancer with ELM by the SCSG/JCOG.

Recently, docetaxel has been considered worth examining for this population. This is because addition of docetaxel to cisplatin and 5-fluorouracil therapy was shown to improve the outcomes of unresectable or recurrent gastric cancer patients [5], although it is not widely accepted as the standard treatment because of its severe toxicity. In Japan, several phase I and phase II trials evaluating combination treatment with docetaxel, cisplatin, and S-1 (DCS) of various regimens with modified doses and schedules for unresectable or recurrent gastric cancer patients have been conducted [6-9]. Although neutropenia and febrile neutropenia occurred frequently, the RR was quite high (76.1-87.7 \%) in each trial. Among the several DCS regimens, we adopted the one used in the phase II trial by Kitasato University (Kitasato regimen) because it was shown to be associated with less toxicity and a higher RR than other regimens, and therefore we considered this DCS regimen to be a promising candidate for the next standard preoperative chemotherapy for ELM. Thus, a multi-institutional phase II trial (JCOG1002) was conducted to evaluate the safety and efficacy of DCS (Kitasato regimen) as preoperative chemotherapy for gastric cancer with ELM.

\section{Methods}

JCOG1002 was conducted as a prospective, multi-institutional, phase II trial by the SCSG/JCOG. All data were handled by the JCOG Data Center. The protocol of this study was approved by the Protocol Review Committee of the JCOG on June 14, 2011 and the institutional review board at each participating center. All enrolled patients provided their written, informed consent. The study was undertaken in accordance with the Declaration of Helsinki and the Japanese Ethical Guidelines for Clinical Studies. The JCOG Data and Safety Monitoring Committee reviewed serious adverse events and judged whether an adverse event was attributable to treatment. This trial was registered with the University Hospital Medical Information Network Clinical Trials Registry (http://www.umin.ac. $\mathrm{jp} / \mathrm{ctr} /$ ) as UMIN000006069. The eligibility criteria are summarized in Table 1. Briefly, patients with gastric cancer with ELM who were suitable candidates for chemotherapy and surgery were enrolled. ELM was defined as PAN metastasis (no. 16a2/16b1) or bulky lymph nodes (one larger than $3 \mathrm{~cm}$ or two larger than $1.5 \mathrm{~cm}$ ) along the celiac, splenic, common, or proper hepatic arteries, or both. The absence of peritoneal dissemination was confirmed by staging laparoscopy before entry into the study. Chest X-ray, contrast-enhanced thoracic/abdominal/ pelvic computed tomography (CT), upper gastrointestinal tract endoscopy, and staging laparoscopy were conducted as the pretreatment workup. 
Table 1 Eligibility criteria

\begin{tabular}{|c|c|}
\hline Inclusion criteria & Exclusion criteria \\
\hline $\begin{array}{l}\text { Histologically proven primary gastric adenocarcinoma } \\
\text { Contrast-enhanced abdominal CT revealed one or both of the } \\
\text { following: PAN metastasis } \geq 1.0 \mathrm{~cm} \text { between the upper margin } \\
\text { of the celiac artery and the upper border of the inferior } \\
\text { mesenteric artery (no. } 16 \mathrm{a} 2 / 16 \mathrm{~b} 1) \text {; bulky lymph nodes } \\
(\geq 3 \mathrm{~cm} \times 1 \text { or } \geq 1.5 \mathrm{~cm} \times 2) \text { along the celiac, splenic, } \\
\text { common, or proper hepatic arteries }\end{array}$ & $\begin{array}{l}\text { Synchronous or metachronous (within } 5 \text { years) malignancies other than } \\
\text { carcinoma in situ or mucosal carcinoma } \\
\text { Pregnant or breastfeeding women } \\
\text { Severe mental disease } \\
\text { Currently treated with systemic steroids } \\
\text { Hepatitis B surface antigen positive }\end{array}$ \\
\hline $\begin{array}{l}\text { Contrast-enhanced thoracic/abdominal/pelvic CT revealed none } \\
\text { of the following: mediastinal lymph node metastasis; lung } \\
\text { metastasis; peritoneal metastasis; liver metastasis; pleural } \\
\text { effusion, ascites; para-aortic lymph node metastasis other than } \\
\text { no. 16a2/16b1; other distant metastases }\end{array}$ & $\begin{array}{l}\text { Currently treated with flucytosine, phenytoin, or warfarin } \\
\text { Iodine allergy } \\
\text { History of hypersensitivity to docetaxel, cisplatin, or polysorbate } 80 \\
\text { Peripheral motor neuropathy or peripheral sensory neuropathy for any }\end{array}$ \\
\hline $\begin{array}{l}\text { The macroscopic tumor type is neither Borrmann type } 4 \text { nor } \\
\text { large }(\geq 8 \mathrm{~cm}) \text { type } 3\end{array}$ & $\begin{array}{l}\text { reason } \\
\text { Edema of the limbs and trunk for any reason }\end{array}$ \\
\hline No esophageal invasion or invasion of $3 \mathrm{~cm}$ or less & Interstitial pneumonia, pulmonary fibrosis or severe emphysema \\
\hline No gastric stump cancer & Active bacterial or fungal infections \\
\hline No clinical signs of cervical lymph node or distant metastases & $\begin{array}{l}\text { History of myocardial infarction or unstable angina pectoris within } \\
6 \text { months }\end{array}$ \\
\hline $\begin{array}{l}\text { A staging laparoscopy or laparotomy performed within } 28 \text { days } \\
\text { revealed negative washing cytology findings and no peritoneal } \\
\text { metastasis }\end{array}$ & $\begin{array}{l}\text { Uncontrolled hypertension } \\
\text { Uncontrolled diabetes mellitus or routine administration of insulin }\end{array}$ \\
\hline \multicolumn{2}{|l|}{ Aged between 20 and 75 years } \\
\hline \multicolumn{2}{|l|}{ An ECOG performance status of 0 or 1} \\
\hline \multicolumn{2}{|l|}{$\begin{array}{l}\text { No prior chemotherapy, radiotherapy, or endocrine therapy for } \\
\text { any malignancies }\end{array}$} \\
\hline \multicolumn{2}{|l|}{$\begin{array}{l}\text { No prior surgery for gastric carcinoma except bypass surgery and } \\
\text { endoscopic resection }\end{array}$} \\
\hline \multicolumn{2}{|l|}{ Fair oral intake with or without bypass surgery } \\
\hline \multicolumn{2}{|l|}{$\begin{array}{l}\text { Adequate organ function: WBC count } \geq 4000 / \mathrm{mm}^{3} \\
\text { and } \leq 12,000 / \mathrm{mm}^{3} ; \text { neutrophil count } \geq 2000 / \mathrm{mm}^{3} ; \\
\text { hemoglobin } \geq 8.0 \mathrm{~g} / \mathrm{dL} ; \text { platelet count } \geq 100,000 / \mathrm{mm}^{3} ; \\
\text { AST and ALT } \leq 100 \mathrm{IU} / \mathrm{L} ; \text { total bilirubin } \leq 1.5 \mathrm{mg} / \mathrm{dL} ; \\
\text { creatinine } \leq 1.2 \mathrm{mg} / \mathrm{dL} \text { and creatinine clearance } \geq 60 \mathrm{~mL} / \mathrm{min}\end{array}$} \\
\hline Written informe & \\
\hline
\end{tabular}

$A L T$ alanine aminotransferase, AST aspartate aminotransferase, $C T$ computed tomography, ECOG Eastern Cooperative Oncology Group, PAN para-aortic lymph node, $W B C$ white blood cell

\section{Preoperative chemotherapy}

Patients received an infusion of docetaxel $\left(40 \mathrm{mg} / \mathrm{m}^{2}\right)$ and cisplatin $\left(60 \mathrm{mg} / \mathrm{m}^{2}\right)$ on day 1 , and orally took $\mathrm{S}-1(40 \mathrm{mg} /$ $\mathrm{m}^{2}$, twice daily) for 2 weeks on days $1-14$, followed by a 2-week rest. Patients were assessed every week during preoperative chemotherapy, but the assessments on days 8 and 22 could be omitted at the discretion of the physician responsible for the patients. The baseline assessment included physical examination and laboratory tests. Toxicity was assessed according to the Common Terminology Criteria for Adverse Events, version 4.0 [10]. The subsequent chemotherapy cycle was delayed until patient recovery, which included the following parameters: neutrophil count of $1500 / \mathrm{mm}^{3}$ or higher, hemoglobin concentration of $8.0 \mathrm{~g} / \mathrm{dL}$ or higher, platelet count of $75,000 /$ $\mathrm{mm}^{3}$ or higher, aspartate aminotransferase and alanine aminotransferase levels of $100 \mathrm{IU} / \mathrm{L}$ or lower, total serum bilirubin level of $2.0 \mathrm{mg} / \mathrm{dL}$ or lower, and serum creatinine concentration of $1.5 \mathrm{mg} / \mathrm{dL}$ or lower. Nonhematological adverse events of fatigue, diarrhea, allergic reaction, pneumonitis, peripheral neuropathy, and hearing impairment were required to be grade 0 or grade 1 . The docetaxel, cisplatin, and S-1 doses were reduced in the event of grade 4 neutropenia, grade 3 or grade 4 thrombocytopenia, total serum bilirubin level greater than $3.0 \mathrm{mg} / \mathrm{dL}$, serum creatinine concentration greater than $1.5 \mathrm{mg} / \mathrm{dL}$, a grade 3 nonhematological adverse event such as febrile neutropenia, nausea, vomiting, anorexia, fatigue, increased alkaline phosphatase level, increased $\gamma$-glutamyltransferase level, hyperglycemia, hypercalcemia, hypocalcemia, hypernatremia, hyponatremia, hyperkalemia, or hypokalemia, or a 
grade 2 or greater nonhematological adverse event such as diarrhea, stomatitis, rash maculopapular, palmar-plantar erythrodysesthesia syndrome, urticaria, allergic reaction, peripheral motor neuropathy, peripheral sensory neuropathy, or hearing impairment. Between day 22 and day 28 of every cycle of preoperative chemotherapy, efficacy was evaluated on the basis of contrast-enhanced CT findings and tumor marker levels. Two cycles of preoperative chemotherapy were performed unless obvious progression or unacceptable adverse events were observed. The possibility of R0 resection was evaluated after the second cycle or immediately after discontinuation of chemotherapy. If $\mathrm{R} 0$ resection was considered possible, the patient underwent surgery within 56 days after the last administration of $\mathrm{S}-1$. If R0 resection was considered difficult despite tumor shrinkage after the second cycle, a third cycle of DCS therapy could be added before surgery. If curative resection was considered difficult without any shrinkage of metastases, the protocol treatment was terminated.

\section{Surgery}

Patients underwent surgery if R0 resection was considered possible on the basis of the findings of the CT scan after the last treatment with $\mathrm{S}-1$, and if they showed adequate organ functions on laboratory tests within 14 days before surgery, which included the following parameters: white blood cell count of $3000 / \mathrm{mm}^{3}$ or greater, platelet count of $100,000 /$ $\mathrm{mm}^{3}$ or greater, aspartate aminotransferase and alanine aminotransferase levels of $100 \mathrm{IU} / \mathrm{L}$ or lower, total serum bilirubin level of $2.0 \mathrm{mg} / \mathrm{dL}$ or lower, and serum creatinine concentration of $1.5 \mathrm{mg} / \mathrm{dL}$ or lower. Nonhematological adverse events of fatigue, diarrhea, and pneumonitis were required to be grade 0 or grade 1. After laparotomy, resectability was reevaluated, and intraperitoneal washing cytology specimens were examined. Briefly, $100 \mathrm{~mL}$ of saline was introduced into the pouch of Douglas and aspirated after gentle stirring. If the cytology findings were negative, R0 resection was attempted by D2 gastrectomy with PAN dissection, as described previously [11]. Laparoscopic surgery, thoracotomy for lower esophageal resection or nodal dissection, left upper abdominal exenteration, pancreaticoduodenectomy, or the so-called Appleby operation was not allowed. Even when distant metastases were found during surgery, surgery with metastasectomy was allowed as long as R0 resection was considered possible. If $\mathrm{R} 0$ resection was considered impossible, the protocol treatment was terminated.

\section{Postoperative chemotherapy}

S-1 therapy was to be started within 42 days of surgery if patients had undergone R0 resection pathologically.
According to the Japanese gastric cancer treatment guidelines from 2010 (third edition) [12], a 6-week cycle consisting of 4 weeks of oral administration of S-1 at a dosage of $40 \mathrm{mg} / \mathrm{m}^{2}$ twice daily followed by 2 weeks of rest was repeated during the first year after surgery. Even after R0 resection, if the tumor progressed during preoperative chemotherapy and histological examination of the resected specimen showed no chemotherapeutic effect, the protocol treatment was terminated, and S-1 was not administered. If S-1 therapy was not started within 12 weeks after surgery for any reason, protocol treatment was terminated. The protocol treatment was completed when a patient had received two or three courses of preoperative chemotherapy, had undergone R0 resection by gastrectomy with D2 plus PAN dissection, and had received 1 year of postoperative chemotherapy. After completion of the protocol, no further treatment was given until tumor recurrence.

\section{Follow-up}

All enrolled patients were to be followed up for 5 years. Efficacy and safety were to be evaluated at least every 3 months until the third year, then at least every 6 months for the last 2 years. General condition and blood tests were checked every 3 months until the third year and every 6 months for the last 2 years. Abdominal CT was performed every 6 months until the third year and every year for the last 2 years. Chest X-ray and upper gastrointestinal tract endoscopy were performed each year.

\section{Study design and statistical analysis}

This trial was designed to evaluate the efficacy and safety of preoperative DCS therapy followed by gastrectomy with D2 plus PAN dissection followed by postoperative S-1 therapy. Although the clinical RR was evaluated by the physician in charge at each institution, to compare the results with those of our previous phase II studies, the primary end point was the clinical RR evaluated by central peer review according to the Response Evaluation Criteria in Solid Tumors version 1.0 [13] for all eligible patients. The secondary end points were 3-year survival, 5-year survival, proportion of R0 resection, proportion of completion of surgery, proportion of completion of adjuvant chemotherapy, pathological RR, and adverse events. Pathological response was evaluated and graded by the institutional pathologists according to the Japanese classification of gastric carcinoma, third English edition [14]: grade $1 \mathrm{a}$, viable tumor cells remain in more than two thirds of the tumorous area; grade $1 \mathrm{~b}$, more than one third but less than two thirds; grade 2, less than one third; grade 3, no viable tumor cells. In this study, pathological response was defined as grade $1 \mathrm{~b}$ to grade 3 response [3]. If this regimen 
proves promising, the next phase III trial is designed to evaluate the superiority of preoperative DCS therapy to preoperative CS therapy in terms of overall survival. The RR was $64.7 \%$ in JCOG0405, and the efficacy of DCS therapy was expected to be superior to that of CS therapy in JCOG0405. In this phase II trial, the sample size was calculated to be 50 cases, under the hypothesis that the expected RR and threshold RR were 80 and $65 \%$, respectively, with use of one-sided testing at the $10 \%$ significance level with power of $80 \%$.

\section{Results}

Between July 2011 and May 2013, 53 patients from 22 institutions entered the study. Table 2 shows the patient demographics and tumor characteristics; $73.6 \%$ of patients had bulky lymph nodes, and $43.4 \%$ had PAN metastases. One patient was not eligible because of lack of target bulky lymph nodes judged by the central peer review. This patient was not included in the efficacy analysis but was included in the safety analysis (Fig. 1).

\section{Preoperative chemotherapy}

There were no complete responses, partial responses in 30 patients, stable diseases in 17 patients, and progressive diseases in two patients. Three patients were considered not evaluable on central peer review. In the two patients who were considered to be not evaluable, preoperative chemotherapies were terminated after the first cycle because of an adverse event and tumor progression respectively. Another patient refused protocol treatment, including CT scan, after the second cycle. The clinical RR for all 52 eligible patients (primary end point) was $57.7 \%$ (80 \% confidence interval 47.9-67.1\%, $95 \%$ confidence interval 43.2-71.3\%). The null hypothesis was not rejected (one-sided $p=0.89$ ). Dose reductions were required once in eight patients $(15.1 \%)$ and twice in one patient $(1.9 \%)$ during preoperative chemotherapy. The mean percentages of the actual/planned dose of docetaxel, cisplatin, and S-1 in each patient were 97.1, 96.7, and 93.2\% respectively. The clinical RRs were 50.0 and $59.1 \%$ with and without dose reduction respectively.

\section{Surgical and pathological findings}

Of the 52 eligible patients, 48 patients underwent surgery. Three patients had unresectable disease and consequently underwent exploratory laparotomy. R0 resection was achieved in 44 patients, whereas the intervention was classified R2 in four cases (Fig. 1). The R0 resection rate was $84.6 \%$ (44/52). The completion proportion of
Table 2 Patient $(n=53)$ and tumor characteristics

\begin{tabular}{|c|c|}
\hline & Values \\
\hline Age (years) ${ }^{\mathrm{a}}$ & $63(38-75)$ \\
\hline Sex ratio (male/female) & $41: 12$ \\
\hline \multicolumn{2}{|l|}{ ECOG performance status } \\
\hline 0 & $48(91 \%)$ \\
\hline 1 & $5(9 \%)$ \\
\hline \multicolumn{2}{|c|}{ Macroscopic type (Japanese classification) } \\
\hline 0 & $2(4 \%)$ \\
\hline 1 & $3(6 \%)$ \\
\hline 2 & $22(42 \%)$ \\
\hline 3 & $22(42 \%)$ \\
\hline 5 & $4(8 \%)$ \\
\hline \multicolumn{2}{|l|}{ Histological type } \\
\hline Intestinal & $29(55 \%)$ \\
\hline Diffuse & $24(45 \%)$ \\
\hline \multicolumn{2}{|l|}{ Tumor depth } \\
\hline $\mathrm{cT} 1$ & 0 \\
\hline $\mathrm{cT} 2$ & $2(4 \%)$ \\
\hline cT3 & $11(21 \%)$ \\
\hline cT4 & $40(75 \%)$ \\
\hline \multicolumn{2}{|l|}{ Lymph node metastases } \\
\hline $\mathrm{cN} 1$ & $2(4 \%)$ \\
\hline $\mathrm{cN} 2$ & $36(68 \%)$ \\
\hline $\mathrm{cN} 3 \mathrm{a}, \mathrm{cN} 3 \mathrm{~b}$ & $15(28 \%)$ \\
\hline \multicolumn{2}{|l|}{ M category } \\
\hline $\mathrm{cM} 0$ & $30(57 \%)$ \\
\hline $\mathrm{cM} 1^{\mathrm{b}}$ & $23(43 \%)$ \\
\hline \multicolumn{2}{|l|}{ cStage } \\
\hline IA & 0 \\
\hline IB & 0 \\
\hline IIA & $1(2 \%)$ \\
\hline IIB & 0 \\
\hline IIIA & $9(17 \%)$ \\
\hline IIIB & $15(28 \%)$ \\
\hline IIIC & $5(9 \%)$ \\
\hline IV & $23(43 \%)$ \\
\hline \multicolumn{2}{|l|}{ Node status } \\
\hline Bulky lymph nodes only & $30(57 \%)$ \\
\hline PAN only & $14(26 \%)$ \\
\hline PAN and bulky lymph nodes & $9(17 \%)$ \\
\hline
\end{tabular}

TNM categories are based on 14th Japanese classification of gastric carcinoma (corresponding to the third English edition)

ECOG Eastern Cooperative Oncology Group, PAN para-aortic lymph node

a The median is given, with the range in parentheses.

b Including PAN metastases

preoperative chemotherapy and surgery was $80.8 \%$ (42/ 52). The surgical data are summarized in Table 3. Seven cases $(15.2 \%)$ were staged pathologically as pM1, most of 
Fig. 1 Patient flowchart

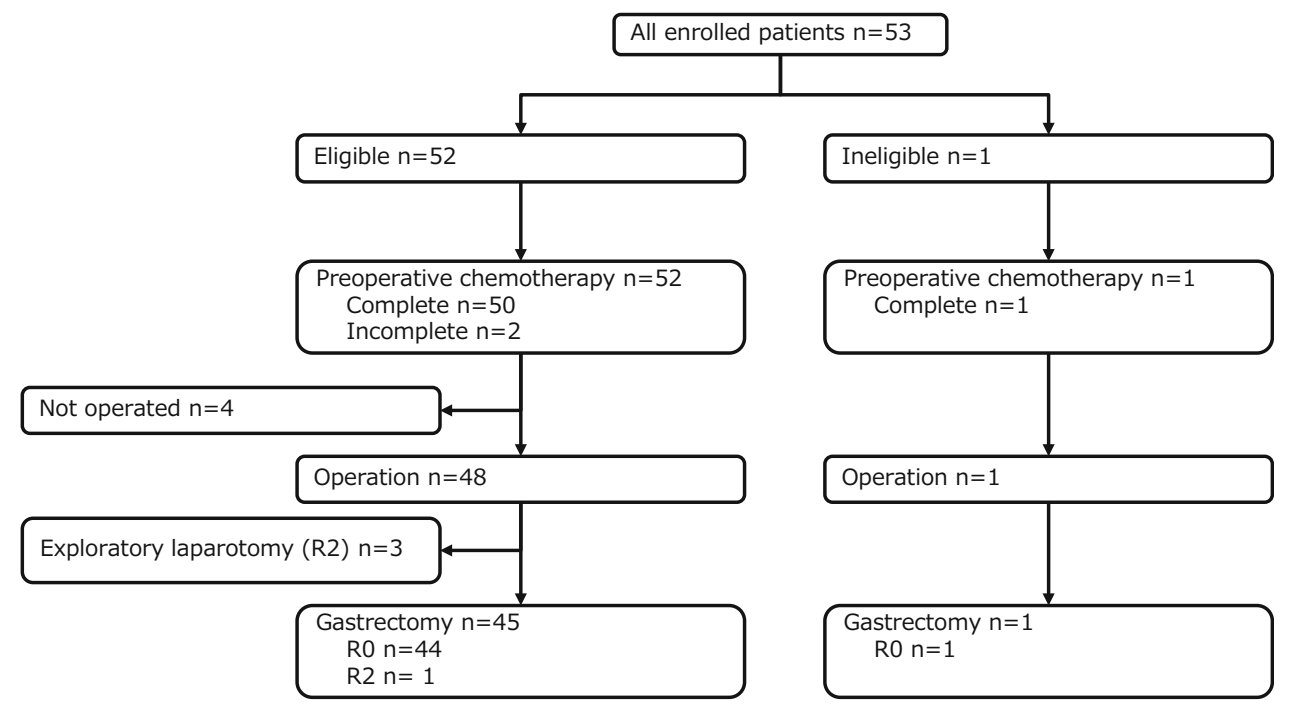

Table 3 Surgical findings in 49 operated on patients

\begin{tabular}{ll}
\hline & Values \\
\hline Tumor location & \\
Upper & $15(31 \%)$ \\
Middle & $20(41 \%)$ \\
Lower & $14(29 \%)$ \\
Gastrectomy & \\
Distal & $15(31 \%)$ \\
Total & $31(63 \%)$ \\
Not resected & $3(6 \%)$ \\
Lymph node dissection & \\
D0 & $3(6 \%)$ \\
D1+ & $1(2 \%)$ \\
D2+ & $45(92)$ \\
Operation time $(\min )^{\mathrm{a}}$ & $402(95-666)$ \\
Blood loss (mL) & $888(10-5428)$ \\
Blood transfusion & \\
Yes & $15(31 \%)$ \\
No & $34(69 \%)$ \\
\hline
\end{tabular}

${ }^{a}$ The median value is given, with the range in parentheses

which were PAN metastases (Table 4). According to the initial clinical node status in 46 patients (including one ineligible patient) who underwent gastrectomy, PAN metastasis was found histologically in 2 of 27 patients with bulky lymph node disease alone, in 3 of 11 patients with PAN involvement alone, and in 2 of 8 patients with both bulky lymph nodes and PAN-positive tumors. The pathological RR for primary tumors in eligible patients was $50.0 \%(26 / 52)$, including one complete remission. Pathological response was seen in only $12.5 \%$ of patients with dose reduction; in contrast, it was seen in $56.8 \%$ of patients without dose reduction.

\section{Adverse events from chemotherapy and surgical complications}

Grade 3 or grade 4 toxicity during preoperative chemotherapy among all 53 treated patients included leukopenia (18.9\%), neutropenia (39.6\%), anemia $(7.5 \%)$, thrombocytopenia (1.9\%), hyponatremia $(15.1 \%)$, hypokalemia $(5.7 \%)$, anorexia $(9.4 \%)$ diarrhea $(7.5 \%)$, nausea $(1.9 \%)$, febrile neutropenia $(5.7 \%)$, and upper respiratory tract infection $(1.9 \%)$. The incidence of grade 3 or grade 4 nonhematological adverse events, excluding adverse events on the basis of laboratory tests, was $20.8 \%$. There were no chemotherapy-related deaths. Morbidity after surgery is summarized in Table 5. Sixteen grade 3 or grade 4 adverse events occurred in 15 patients $(30.6 \%)$. Any grade of pancreatic fistula, intra-abdominal abscess, or anastomotic leakage occurred in 18.4, 10.2, and $4.1 \%$ of patients respectively. Two reoperations were performed because of anastomotic leakage and small intestinal obstruction. Although one in-hospital death resulted from disseminated intravascular coagulation caused by rapid tumor progression, the JCOG Data and Safety Monitoring Committee judged that this early death was not related to the protocol treatment. No treatmentrelated deaths occurred.

\section{Discussion}

The present multi-institutional phase II trial of preoperative chemotherapy showed acceptable safety and an equivalent R0 resection rate as our previous study (82\% in JCOG0405) [4], but it did not show sufficient efficacy in terms of tumor response. The eligibility criteria and multimodality treatment, except for the regimen of 
Table 4 Pathological findings for 46 patients

\begin{tabular}{|c|c|}
\hline & Values \\
\hline \multicolumn{2}{|l|}{ Tumor depth } \\
\hline урТ0 & $1(2 \%)$ \\
\hline урT1 & $8(17 \%)$ \\
\hline урT2 & $9(20 \%)$ \\
\hline урТ3 & $21(46 \%)$ \\
\hline ypT4 & $7(15 \%)$ \\
\hline \multicolumn{2}{|l|}{ Lymph node metastases } \\
\hline ypNO & $10(22 \%)$ \\
\hline ypN1 & $8(17 \%)$ \\
\hline ypN2 & $9(20 \%)$ \\
\hline ypN3a, ypN3b & $19(41 \%)$ \\
\hline \multicolumn{2}{|l|}{ PAN metastases } \\
\hline No & $37(80 \%)$ \\
\hline Yes & $7(15 \%)$ \\
\hline Not evaluated & $2(4 \%)$ \\
\hline $\begin{array}{l}\text { Median number of retrieved lymph nodes } \\
(\mathrm{D} 1+\mathrm{D} 2+\mathrm{PAN})^{\mathrm{a}}\end{array}$ & 68 \\
\hline Median number of retrieved lymph nodes $(\mathrm{PAN})^{\mathrm{a}}$ & $18(3-55)$ \\
\hline \multicolumn{2}{|l|}{ Peritoneal disease } \\
\hline No & $45(98 \%)$ \\
\hline Yes & $1(2 \%)$ \\
\hline \multicolumn{2}{|l|}{ M category } \\
\hline M0 & $39(85 \%)$ \\
\hline ypM1 $1^{\text {b }}$ & $7(15 \%)$ \\
\hline \multicolumn{2}{|l|}{ Resection } \\
\hline R0 & $45(98 \%)$ \\
\hline $\mathrm{R} 1$ & $0(0 \%)$ \\
\hline $\mathrm{R} 2$ & $1(2 \%)$ \\
\hline \multicolumn{2}{|l|}{ ypStage } \\
\hline IA & $2(4 \%)$ \\
\hline IB & $7(15 \%)$ \\
\hline IIA & $5(11 \%)$ \\
\hline IIB & $2(4 \%)$ \\
\hline IIIA & $10(22 \%)$ \\
\hline IIIB & $7(15 \%)$ \\
\hline IIIC & $5(11 \%)$ \\
\hline IV & $7(15 \%)$ \\
\hline Not classifiable & $1(2 \%)$ \\
\hline \multicolumn{2}{|l|}{ Histological type } \\
\hline Intestinal & $23(50 \%)$ \\
\hline Diffuse & $21(46 \%)$ \\
\hline Other & $2(4 \%)$ \\
\hline \multicolumn{2}{|l|}{ Lymphatic invasion } \\
\hline No & $22(48 \%)$ \\
\hline Yes & $24(52 \%)$ \\
\hline \multicolumn{2}{|l|}{ Vascular invasion } \\
\hline No & $22(48 \%)$ \\
\hline Yes & $24(52 \%)$ \\
\hline
\end{tabular}

Table 4 continued

\begin{tabular}{ll}
\hline & Values \\
\hline Pathological response & \\
Grade 0 & $3(7 \%)$ \\
Grade 1a & $17(37 \%)$ \\
Grade 1b & $8(17 \%)$ \\
Grade 2 & $17(37 \%)$ \\
Grade 3 & $1(2 \%)$ \\
\hline
\end{tabular}

PAN para-aortic lymph node

a The range is given in parentheses

b Including PAN metastases

Table 5 Morbidity after surgery in 49 patients

\begin{tabular}{lccccc}
\hline & \multicolumn{3}{l}{ Grade $^{\mathrm{a}}$} & & $\begin{array}{l}\text { Grade 3-4 } \\
(\%)\end{array}$ \\
\cline { 2 - 5 } & 1 & 2 & 3 & 4 & \\
\hline Pancreatic fistula & 1 & 5 & 2 & 1 & 6 \\
Abdominal infection & - & - & 5 & 0 & 10 \\
Anastomotic leak & 0 & 1 & 0 & 1 & 2 \\
Anastomotic stricture & 0 & 1 & 0 & 0 & 0 \\
Delayed gastric emptying & 0 & 1 & 0 & 0 & 0 \\
Small intestinal & 0 & 0 & 1 & 0 & 2 \\
$\quad$ obstruction & & & & & \\
Ileus & - & 0 & 1 & 0 & 2 \\
Thromboembolic event & 0 & 0 & 1 & 1 & 4 \\
Lung infection & - & 1 & 3 & 0 & 6 \\
Pleural effusion & 4 & 2 & 0 & 0 & 0 \\
Chylous ascites & 2 & 1 & 0 & 0 & 0 \\
Wound infection & - & 2 & 0 & 0 & 0
\end{tabular}

${ }^{a}$ National Cancer Institute Common Terminology Criteria for Adverse Events version 4.0

preoperative chemotherapy, were essentially the same in the present study and in our previous study. There were no differences in patient and tumor characteristics such as age, sex, macroscopic type, tumor location, node status, and histological type, although Eastern Cooperative Oncology Group performance status was slightly worse in the present study.

One possible explanation for this result is the dose intensity of preoperative chemotherapy. The proportions of febrile neutropenia for the Sapporo [9], Kitasato [7], and Kanazawa [6] regimens were 16.1, 13.5, and $13.3 \%$ respectively, whereas the RRs were 87.1, 81.3, and 76.1\% respectively. Since our previous study (JCOG0001) [3] was terminated early because of treatment-related deaths, the Kitasato regimen was used for its lower toxicity and higher $\mathrm{RR}$ among various DCS regimens. The DCS regimen is intensified treatment, adding docetaxel to the CS regimen, but the dose intensity of S-1 is reduced. Furthermore, in the 
CS regimen of JCOG0405, S-1 was given for the first 3 weeks of a 4-week cycle, in which the dose intensity of S-1 was intensified compared with the CS regimen for unresectable or recurrent gastric cancer. As for S-1 therapy, the dose intensities of CS in JCOG0405 and DCS in the present study were $420 \mathrm{mg} / \mathrm{m}^{2} /$ week and $280 \mathrm{mg} / \mathrm{m}^{2} /$ week respectively. In fact, preoperative chemotherapy with DCS was feasible, and the mean percentage of the actual/planned dose was quite high, although the proportion of PS1 patients was $9 \%$, slightly higher than in our previous study $(0 \%)$. Meanwhile, the incidence of grade 3 or grade 4 febrile neutropenia from DCS therapy in the present study $(5.7 \%)$ was lower than that in the phase II study of the Kitasato regimen for unresectable or recurrent gastric cancer patients (13.6\%) [7]. The proportion of PS0 patients was $90.6 \%$, higher than that for the Kitasato regimen for unresectable or recurrent gastric cancer patients $(67.8 \%)$. The operative patients, who had favorable performance status, might have been able to tolerate more intensive chemotherapy than we had expected.

Another explanation is the number of chemotherapy cycles. In the present study, the number of preoperative chemotherapy cycles was specified in the protocol as only two courses if R0 resection was expected to be possible. The RR of DCS therapy in the phase II study of the Kitasato regimen [7] was high $(81.3 \%)$ for unresectable gastric cancer, but the proportion of patients who underwent DCS therapy and achieved partial response within two cycles among the responders was only $83.3 \%$, which means that the partial response rate within two cycles was only $67.7 \%$ of all patients (personal communication). Thus, the number of chemotherapy cycles might not have been optimized in the present study. Currently, a randomized phase II trial to compare preoperative chemotherapy using two and four cycles of CS therapy and DCS therapy in a two-by-two factorial design is being conducted [15]. Suitable durations of preoperative chemotherapy may be established in the future.

Schulz et al. [16] reported a good response to a neoadjuvant regimen of oxaliplatin, leucovorin, 5-fluorouracil, and docetaxel, predominantly observed in patients with intestinal-type tumors, but there was no difference in efficacy in terms of pathological response between intestinal type and diffuse type ( $48.3 \%$ vs $52.2 \%$ ) in the present study. This discrepancy may be partly due to the difference in fluoropyrimidines. Yamada et al. [17] reported higher dihydropyrimidine dehydrogenase messenger RNA expression in the diffuse type than in the intestinal type. Since S-1 contains gimeracil, which inhibits dihydropyrimidine dehydrogenase activity, the DCS regimen might be effective not only for the intestinal type but also for the diffuse type. Further study is needed to clarify whether the histological type affects the efficacy of docetaxel-containing regimens.
In the present study, D2 plus PAN dissection was mandatory after preoperative chemotherapy. Although PAN dissection in a prophylactic setting has not been shown to be useful [18], we assume that preoperative CS therapy followed by gastrectomy with PAN dissection is the tentative standard treatment for locally advanced gastric cancer with ELM. JCOG0405 showed favorable outcomes (3-year survival of $58.8 \%$ ), although the proportion of pathological N3 including PAN in JCOG0405 was $31 \%$. PAN dissection may have contributed to the favorable outcome. Even in the present study, the proportion of PAN metastases was $15 \%$, indicating that PAN dissection may still be needed for this population. Wang et al. [19] reported preoperative capecitabine and oxaliplatin chemotherapy followed by D2 gastrectomy without PAN dissection for advanced gastric cancer patients with PAN metastases and demonstrated that the median overall survival was 29.8 months, although their follow-up was relatively short. Further study of the role of PAN dissection for tumors with ELM treated by preoperative chemotherapy is needed.

Kurokawa et al. [20] demonstrated that the histological RR seemed to be a better surrogate end point for overall survival than the radiological RR (clinical RR) in studies of neoadjuvant therapy for gastric cancer. Becker et al. [21] showed in their multivariate analysis that the pathological RR with a $10 \%$ cutoff for residual tumor remained a prognostic factor, whereas the proportion of R0 resection did not. Therefore, the pathological RR has become a common end point in preoperative settings in gastric cancer. In the present study, the clinical RR was used as the primary end point because different definitions of the cutoff percentage of residual tumors in histological criteria have impaired the comparability of the results of different trials. Nakamura et al. [22] demonstrated that the $10 \%$ cutoff was the best in terms of the hazard ratio of nonresponders to responders in overall survival and should be the global standard cutoff percentage of residual tumor to determine the pathological RR. In the present study, the incidence of grade 2 or grade 3 (viable tumor cells remain in less than one third of tumorous area) was slightly higher than in our previous CS study (34.6\% vs $28.6 \%$ ), but the RR with the $10 \%$ cutoff was not available. Long-term survival of the patients in the present study will not be known until follow-up is complete, and the efficacy of preoperative DCS chemotherapy should be reevaluated in terms of long-term overall survival.

The limitation of the present study is its single-arm, phase II design. Therefore, a simple comparison of survival with that from our previous study is not justified because there were differences in terms of postoperative adjuvant chemotherapy. A phase III trial is needed to draw a definitive conclusion about the optimal regimen for gastric cancer with ELM. 


\section{Conclusion}

Preoperative DCS therapy was feasible but did not show sufficient efficacy. Preoperative CS therapy is still considered the tentative standard treatment for gastric cancer with ELM until survival results are known.

Acknowledgments The authors thank M. Wakabayashi (Japan Clinical Oncology Group Data Center) and K. Kataoka and K. Nakamura (Japan Clinical Oncology Group Operations Office) as contributors, and M. Miyazawa, A. Higashiohmori, and H. Fukuda (Japan Clinical Oncology Group Data Center) for assistance and support with data collection, running the study, and the analyses.

\section{Compliance with ethical standards}

Funding This study was supported by the National Cancer Center Research and Development Fund (23-A-16, 23-A-19, 26-A-4) and a Health and Labour Sciences Research Grant for Clinical Cancer Research (H22-Gan-016) from the Ministry of Health, Labour and Welfare, Japan.

Conflict of interest Seiji Ito has received grants and personal fees from Chugai Pharmaceutical and Yakult Pharma and personal fees from Covidien, Ely Lilly, Takeda, Nippon Kayaku, and Kyowa Hakko Kirin outside the submitted work. Takeshi Sano received personal fees from Taiho Pharmaceutical during the conduct of the study and has received personal fees from Chugai Pharmaceutical, Yakult Pharma, Ely Lilly, Ethicon, Covidien, Olympus, and Esai outside the submitted work. Hiroaki Onaya has received personal fees from Otsuka Pharmaceutical Co. Ltd outside the submitted work. Mitsuru Sasako has received grants from Taiho Pharmaceutical, Chugai, and Yakult and personal fees from Taiho Pharmaceutical, Chugai, Sanofi, and Yakult outside the submitted work. Junki Mizusawa, Daisuke Takahari, Hiroshi Katayama, Hitoshi Katai, Yoshiyuki Kawashima, Takahiro Kinoshita, Masanori Terashima, Atsushi Nashimoto, and Mikihito Nakamori have nothing to disclose.

Human rights statement and informed consent All procedures followed were in accordance with the ethical standards of the responsible committee on human experimentation (institutional and national) and with the Helsinki Declaration of 1964 and later versions. Informed consent or substitute for it was obtained from all patients for their being included in the study.

\section{References}

1. Ferlay J, Soerjomataram I, Dikshit R, Eser S, Mathers C, Rebelo $\mathrm{M}$, et al. Cancer incidence and mortality worldwide: sources, methods and major patterns in GLOBOCAN 2012. Int J Cancer. 2015;136:E359-86.

2. Park IH, Kim SY, Kim YW, Ryu KW, Lee JH, Lee JS, et al. Clinical characteristics and treatment outcomes of gastric cancer patients with isolated para-aortic lymph node involvement. Cancer Chemother Pharmacol. 2011;67:127-36.

3. Yoshikawa T, Sasako M, Yamamoto S, Sano T, Imamura H, Fujitani K, et al. Phase II study of neoadjuvant chemotherapy and extended surgery for locally advanced gastric cancer. Br J Surg. 2009; 96:1015-22.

4. Tsuburaya A, Mizusawa J, Tanaka Y, Fukushima N, Nashimoto A, Sasako M. Neoadjuvant chemotherapy with S-1 and cisplatin followed by D2 gastrectomy with para-aortic lymph node dissection for gastric cancer with extensive lymph node metastasis. Br J Surg. 2014;101:653-60.

5. Van Cutsem E, Moiseyenko VM, Tjulandin S, Majlis A, Constenla M, Boni C, et al. Phase III study of docetaxel and cisplatin plus fluorouracil compared with cisplatin and fluorouracil as firstline therapy for advanced gastric cancer: a report of the V325 Study Group. J Clin Oncol. 2006;24:4991-7.

6. Fushida S, Fujimura T, Oyama K, Yagi Y, Kinoshita J, Ohta T. Feasibility and efficacy of preoperative chemotherapy with docetaxel, cisplatin and S-1 in gastric cancer patients with para-aortic lymph node metastases. Anticancer Drugs. 2009;20:752-6.

7. Koizumi W, Nakayama N, Tanabe S, Sasaki T, Higuchi K, Nishimura K, et al. A multicenter phase II study of combined chemotherapy with docetaxel, cisplatin, and S-1 in patients with unresectable or recurrent gastric cancer (KDOG 0601). Cancer Chemother Pharmacol. 2012;69:407-13.

8. Nakayama N, Koizumi W, Sasaki T, Higuchi K, Tanabe S, Nishimura K, et al. A multicenter, phase I dose-escalating study of docetaxel, cisplatin and S-1 for advanced gastric cancer (KDOG0601). Oncology. 2008;75:1-7.

9. Sato Y, Takayama T, Sagawa T, Takahashi Y, Ohnuma H, Okubo $\mathrm{S}$, et al. Phase II study of S-1, docetaxel and cisplatin combination chemotherapy in patients with unresectable metastatic gastric cancer. Cancer Chemother Pharmacol. 2010;66:721-8.

10. National Cancer Institute. Common Terminology Criteria for Adverse Events (CTCAE) v4.0. http://ctep.cancer.gov/proto colDevelopment/electronic_applications/ctc.htm\#ctc_40. Accessed 3 June 2016.

11. Sano T, Sasako M, Yamamoto S, Nashimoto A, Kurita A, Hiratsuka $\mathrm{M}$, et al. Gastric cancer surgery: morbidity and mortality results from a prospective randomized controlled trial comparing D2 and extended para-aortic lymphadenectomy-Japan Clinical Oncology Group study 9501. J Clin Oncol. 2004;22:2767-73.

12. Japanese Gastric Cancer Association. Japanese gastric cancer treatment guidelines 2010 (ver. 3). Gastric Cancer. 2011;14:113-23.

13. Therasse P, Arbuck SG, Eisenhauer EA, Wanders J, Kaplan RS, Rubinstein $\mathrm{L}$, et al. New guidelines to evaluate the response to treatment in solid tumors. J Natl Cancer Inst. 2000;92:205-16.

14. Japanese Gastric Cancer Association. Japanese classification of gastric carcinoma: 3rd English edition. Gastric Cancer. 2011;14:101-12.

15. Yoshikawa T, Taguri M, Sakuramoto S, Kunisaki C, Fukunaga T, Ito $\mathrm{S}$, et al. A comparison of multimodality treatment: two and four courses of neoadjuvant chemotherapy using S-1/CDDP or S-1/CDDP/docetaxel followed by surgery and S-1 adjuvant chemotherapy for macroscopically resectable serosa-positive gastric cancer: a randomized phase II trial (COMPASS-D trial). Jpn J Clin Oncol. 2012;42:74-7.

16. Schulz C, Kullmann F, Kunzmann V, Fuchs M, Geissler M, Vehling-Kaiser U, et al. NeoFLOT: multicenter phase II study of perioperative chemotherapy in resectable adenocarcinoma of the gastroesophageal junction or gastric adenocarcinoma-very good response predominantly in patients with intestinal type tumors. Int J Cancer. 2015;137:678-85.

17. Yamada Y, Boku N, Nishina T, Yamaguchi K, Denda T, Tsuji A, et al. Impact of excision repair cross-complementing gene 1 (ERCC1) on the outcomes of patients with advanced gastric cancer: correlative study in Japan Clinical Oncology Group trial JCOG9912. Ann Oncol. 2013;24:2560-5.

18. Sasako M, Sano T, Yamamoto S, Kurokawa Y, Nashimoto A, Kurita A, et al. D2 lymphadenectomy alone or with para-aortic nodal dissection for gastric cancer. N Engl J Med. 2008;359:453-62.

19. Wang Y, Yu YY, Li W, Feng Y, Hou J, Ji Y, et al. A phase II trial of Xeloda and oxaliplatin (XELOX) neo-adjuvant chemotherapy followed by surgery for advanced gastric cancer patients with 
para-aortic lymph node metastasis. Cancer Chemother Pharmacol. 2014;73:1155-61.

20. Kurokawa Y, Shibata T, Sasako M, Sano T, Tsuburaya A, Iwasaki $\mathrm{Y}$, et al. Validity of response assessment criteria in neoadjuvant chemotherapy for gastric cancer (JCOG0507-A). Gastric Cancer. 2014;17:514-21.

21. Becker K, Langer R, Reim D, Novotny A, Meyer zum Buschenfelde C, Engel J, et al. Significance of histopathological tumor regression after neoadjuvant chemotherapy in gastric adenocarcinomas: a summary of 480 cases. Ann Surg. 2011;253:934-9.

22. Nakamura K, Kuwata T, Shimoda T, Mizusawa J, Katayama H, Kushima R, et al. Determination of the optimal cutoff percentage of residual tumors to define the pathological response rate for gastric cancer treated with preoperative therapy (JCOG1004-A). Gastric Cancer. 2015;18:597-604. 